\title{
Joint Channel Estimation and Multiuser Detection for SDMA/OFDM Based on Dual Repeated Weighted Boosting Search
}

\author{
Jiankang Zhang, Student Member, IEEE, Sheng Chen, Fellow, IEEE, Xiaomin Mu, and Lajos Hanzo, Fellow, IEEE
}

\begin{abstract}
A joint channel estimation and multiuser detection (JCEMUD) scheme is proposed for multiuser multipleinput-multiple-output (MIMO) space-division multiple-access/ orthogonal frequency-division-multiplexing (SDMA/OFDM) systems. We design a dual repeated weighted boosting search (DRWBS) scheme for JCEMUD, which is capable of providing "soft" outputs, which are directly fed to the forward error correction (FEC) decoder. The proposed DRWBS-JCEMUD scheme iteratively estimates the channel impulse responses and detects the users' transmitted signals while exploiting the error correction capability of an FEC decoder to iteratively exchange information between the detector and the estimator. Furthermore, the proposed DRWBS-JCEMUD scheme is capable of providing the loglikelihood ratios of the coded bits at low computational complexity (comparable with the single-user scenario), which can directly be fed to the FEC decoder. The simulation results demonstrate that the proposed DRWBS-JCEMUD scheme is capable of attaining a mean square error performance close to that of the ideal scenario of the least-square channel estimator associated with $100 \%$ pilot overhead and narrows the discrepancy with respect to the optimal maximum-likelihood (ML) MUD associated with perfect channel knowledge. As an example, at $E_{b} / N_{0}=10 \mathrm{~dB}$, a factor-of- 0.756 complexity reduction was achieved at the cost of a 1-dB performance penalty, in comparison with the ML-MUD.
\end{abstract}

Index Terms-Joint channel estimation and multiuser detection (JCEMUD), orthogonal frequency-division multiplexing (OFDM), repeated weighted boosting search (RWBS), space-division multiple access (SDMA).

\section{INTRODUCTION}

C OMMUNICATION systems using multiple antennas at the transmitter and/or the receiver have recently received increased attention due to their ability to provide substantial capacity improvements while achieving low error rate and/or

Manuscript received February 22, 2011; revised May 30, 2011; accepted June 24, 2011. Date of publication July 7, 2011; date of current version September 19, 2011. This work was supported by the China Scholarship Council and the European Union under the auspices of the OPTIMIX project, as well as of the Research Council UK under the India-UK Advanced Technology Center Initiative. The review of this paper was coordinated by Dr. H. Lin.

J. Zhang was with the School of Electronics and Computer Science, University of Southampton, SO17 1BJ Southampton, U.K. He is now with the School of Information Engineering, Zhengzhou University, Zhengzhou 450001, China (e-mail: jankyzhang@yeah.net).

S. Chen and L. Hanzo are with the School of Electronics and Computer Science, University of Southampton, SO17 1BJ Southampton, U.K. (e-mail: sqc@ecs.soton.ac.uk; 1h@ecs.soton.ac.uk).

$\mathrm{X} . \mathrm{Mu}$ is with the School of Information Engineering, Zhengzhou University, Zhengzhou 450001, China (e-mail: iexmmu@zzu.edu.cn).

Color versions of one or more of the figures in this paper are available online at http://ieeexplore.ieee.org.

Digital Object Identifier 10.1109/TVT.2011.2161356 high data rate by flexibly exploiting the attainable diversity gain and/or the spatial multiplexing gain [1].

Space-division multiple-access/orthogonal frequencydivision-multiplexing (SDMA/OFDM) systems [2], [3] combine the advantages of OFDM and SDMA, where we employ an array of antennas at the base station (BS) for detecting the received signal of multiple single-antenna aided mobile stations (MSs). As a result, a substantially improved uplink (UL) capacity is achieved, despite employing single-antennabased low-complexity MS transmitters [3]. However, the performance of these systems is critically dependent on the precision of the channel knowledge, which may be represented by either the channel impulse response (CIR) or the FrequencyDomain CHannel Transfer Function (FD-CHTF).

Over the past decade, intensive research efforts have been devoted to developing effective approaches for both channel estimation and multiuser symbol detection for transmitterand/or receiver-diversity-aided systems [3], [4]. Conventional methods usually carry out the channel estimation and signal detection separately, which may only attain suboptimal results. To achieve near-optimal performance, joint channel estimation and data detection algorithms have recently received significant research attention [4]-[7]. These joint channel estimation and data detection methods have indeed shown an enhanced performance associated with reasonable convergence rates despite using relatively short pilot symbol sequences. Among them, the iterative expectation-maximization (EM) algorithm [8] and the diverse derivatives of this algorithm have been shown to strike an attractive tradeoff between the performance attained and the complexity imposed. The classic EM algorithm was employed for joint channel estimation and data detection in [7] and [9]. The authors of [10] proposed a joint symbol detection and channel estimation algorithm based on the variational Bayesian EM algorithm. A space-alternating generalized EM-based iterative receiver was designed for joint detection, decoding, and channel estimation in [11]. However, the EM algorithm is unable to guarantee convergence to the globally optimal solution. Furthermore, genetic algorithm (GA)-based near-optimal search schemes were also developed for channel estimation and data symbol detection at the receiver [5], [12], [13]. Finally, in [14], repeated weighted boosting search (RWBS) was employed to identify the unknown multiple-input-multiple-output (MIMO) channel, while an enhanced maximum-likelihood (ML) sphere detector was used to perform ML detection of the transmitted data. 


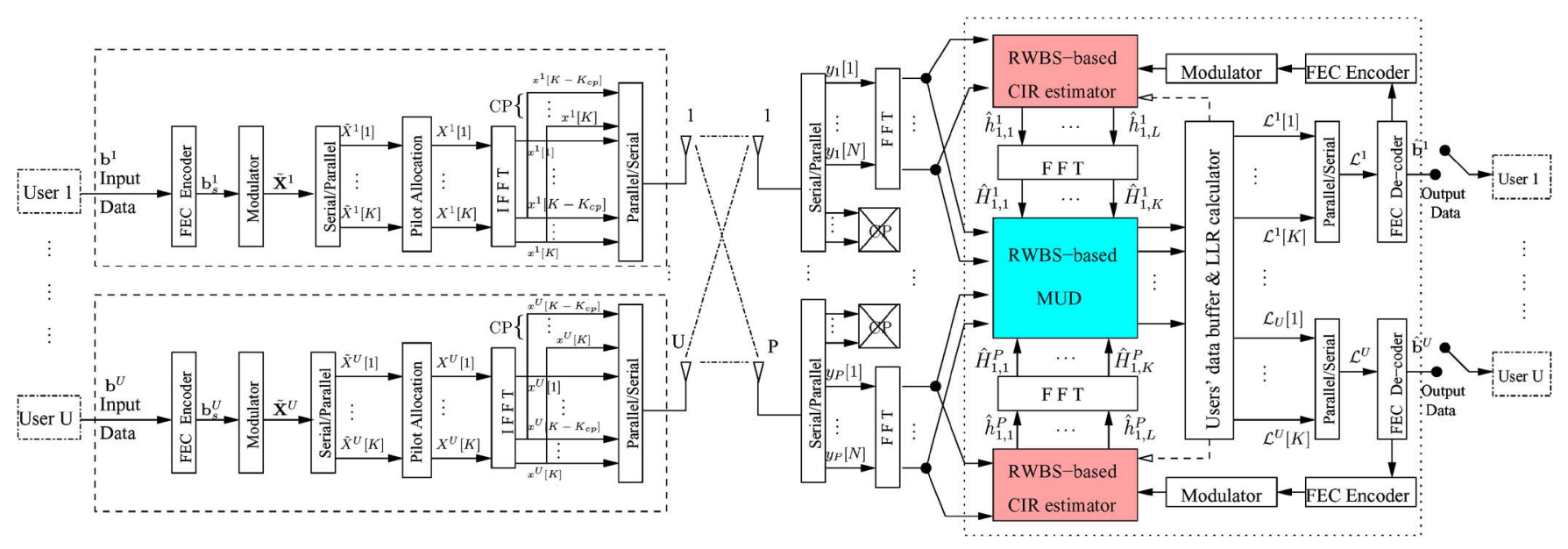

Fig. 1. Uplink system model for multiuser MIMO SDMA/OFDM.

Against this background, in this paper, we proposed a novel guided random search algorithm, which we refer to as the dual RWBS (DRWBS) assisted joint channel estimation and multiuser detection (DRWBS-JCEMUD) scheme designed for multiuser MIMO SDMA/OFDM systems. The proposed DRWBS-JCEMUD scheme consists of two components, namely, the RWBS-based channel estimator and the RWBSbased multiuser detector (MUD). The channel estimator carries out channel estimation using the available detected symbols, whereas the symbol detector carries out symbol detection using the available channel estimate. The process is carried out by iteratively exchanging information between the channel estimator and the symbol detector. Furthermore, the proposed DRWBS-JCEMUD scheme is capable of providing the loglikelihood ratios (LLRs) of the coded bits, which can directly be fed to the forward error correction (FEC) decoder.

The rest of this paper is organized as follows: The system model of the multiuser MIMO SDMA/OFDM UL is described in Section II. The proposed DRWBS-JCEMUD scheme is elaborated upon in Section III. In Section IV, we analyze the computational complexity of the proposed DRWBS-JCEMUD scheme, followed by its convergence characterization. Our simulation results and discussions are presented in Section V, whereas our conclusions are offered in Section VI.

\section{SYSTEM MODEL}

The multiuser MIMO SDMA/OFDM system considered supports $U$ MSs simultaneously transmitting in the UL to the BS, as shown in Fig. 1. Each of the users is equipped with a single transmit antenna, whereas the BS employs an array of $P$ antennas. It is assumed that a time-division multiple-access protocol organizes the division of the available time-domain (TD) resources into SDMA/OFDM time slots. Instead of one, $U$ MSs are assigned to each slot that is allowed to simultaneously transmit their streams of OFDM-modulated symbols to the SDMA BS [2], [3].

More specially, all of the $U$ MSs transmit independent data streams, which are encoded by a user-specific FEC encoder, as illustrated in Fig. 1. The information bits output by the FEC encoder are grouped and mapped to a stream of modulated data symbols. The modulated data $\mathbf{X}^{(u)}[k], k=1,2, \ldots, K$ in Fig. 1 are then serial to parallel converted, and the frequencydomain training symbols are concatenated at the beginning of each frame. The parallel modulated data are further processed by the inverse fast Fourier transform (FFT) to form a set of OFDM symbols. After concatenating the cyclic prefix (CP) of $K_{\mathrm{cp}}$ samples, the TD signal is transmitted through a multipath fading channel and contaminated by the receiver's additive white Gaussian noise (AWGN).

At the BS, the CP is discarded from every OFDM symbol, and the resultant signal is fed into the corresponding FFT-based receiver in Fig. 1. Let $Y_{p}[s, k]$ denote the signal received by the $p$ th receiver antenna element in the $k$ th subcarrier of the sth OFDM symbol, which is given as the superposition of the different users' channel-impaired received signal contributions plus the AWGN, which is expressed as [3]

$$
Y_{p}[s, k]=\sum_{u=1}^{U} H_{p}^{u}[s, k] X^{u}[s, k]+W_{p}[s, k]
$$

where $H_{p}^{u}[s, k]$ denotes the FD-CHTF of the link between the $u$ th user and the $p$ th receiver antenna in the $k$ th subcarrier of the sth OFDM symbol.

As a benefit of the CP, the SDMA/OFDM symbols do not overlap, and hence, SDMA processing can be applied on a percarrier basis, as depicted in Fig. 1. Each SDMA subcarrier's signal may be described as [2]

$$
\begin{aligned}
{\left[\begin{array}{c}
\tilde{X}^{1}[s, k] \\
\vdots \\
\tilde{X}^{U}[s, k]
\end{array}\right]=\mathcal{F}_{S D M A} } & \left(\left[\begin{array}{c}
Y_{1}[s, k] \\
\vdots \\
Y_{P}[s, k]
\end{array}\right],\right. \\
& {\left.\left[\begin{array}{ccc}
\hat{H}_{1}^{1}[s, k] & \ldots & \hat{H}_{1}^{U}[s, k] \\
\vdots & \ldots & \vdots \\
\hat{H}_{P}^{1}[s, k] & \ldots & \hat{H}_{P}^{U}[s, k]
\end{array}\right]\right) }
\end{aligned}
$$

where $\mathcal{F}_{S D M A}(\cdot)$ represents a SDMA subprocessor, which is constituted by the MUD. In addition, $\hat{H}_{p}^{u}[s, k], u=1, \ldots, U$, $p=1, \ldots, P$, is the estimated value of the FD-CHTF. Various algorithmic alternatives for this generic SDMA detector are available, which cover a wide range of performance 
versus complexity tradeoffs, such as those represented by the minimum mean square error (MMSE) SDMA/OFDM, per-carrier successive interference cancellation SDMA/OFDM, and ML SDMA/OFDM [2]. In the following section, we outline our DRWBS-JCEMUD scheme designed for multiuser MIMO SDMA/OFDM systems.

\section{DRWBS-JCEMUD SCHEME PROPOSED FOR MULTIUSER MIMO SDMA/OFDM SYSTEMS}

\section{A. JCEMUD}

In the context of the JCEMUD problem of the SDMA/OFDM systems, the cost function (CF) can be based on the loglikelihood function conditioned both on the matrix $\mathbf{h}[s]$ containing the CIR coefficients and on the users' transmitted data $\mathbf{X}[s]$ incorporating the $M$-quadrature amplitude modulated (QAM) signals, which is given by

$$
J(\mathbf{h}[s], \mathbf{X}[s])=\sum_{p=1}^{P}\left\|\mathbf{Y}_{p}[s]-\mathbf{X}^{T}[s] \mathbf{F} \mathbf{h}_{p}[s]\right\|^{2}
$$

where the received data $\mathbf{Y}_{p}[s] \in \mathbb{C}^{K \times 1}$, the transmitted data $\mathbf{X}[s] \in \mathbb{C}^{U K \times K}$, the block diagonal matrix $\mathbf{F} \in \mathbb{C}^{U K \times U L}$, and the CIRs $\mathbf{h}_{p}[s] \in \mathbb{C}^{U L \times 1}$ in (3) are defined as

$$
\begin{aligned}
\mathbf{Y}_{p}[s] & =\left[Y_{p}[s, 1], Y_{p}[s, 2], \ldots, Y_{p}[s, K]\right]^{T} \\
\mathbf{X}[s] & =\left[\mathbf{X}^{1}[s], \mathbf{X}^{2}[s], \ldots, \mathbf{X}^{U}[s]\right]^{T} \\
\mathbf{X}^{u}[s] & =\operatorname{diag}\left\{X^{u}[s, 1], X^{u}[s, 2], \ldots, X^{u}[s, K]\right\} \\
\mathbf{F} & =\operatorname{diag}\left\{\mathbf{F}^{1}, \mathbf{F}^{2}, \ldots, \mathbf{F}^{U}\right\} \\
\mathbf{h}_{p}[s] & =\left[\mathbf{h}_{p}^{1}[s], \mathbf{h}_{p}^{2}[s], \ldots, \mathbf{h}_{p}^{U}[s]\right]^{T} .
\end{aligned}
$$

Furthermore, $\mathbf{F}^{u}, u=1,2, \ldots, U$, in (7) is a $(K \times L)$ element FFT matrix with $F^{u}[k, l]=e^{-j 2 \pi(k-1)(l-1) / K}, 1 \leq$ $k \leq K, 1 \leq l \leq L$.

The ML problem is equivalent to a least-square problem since the received data are corrupted by the Gaussian noise, which can then be viewed as a special case of a convex optimization problem [15] (see [15] and references therein for more detailed discussions on convex optimization). In [16], a Viterbi-algorithm-aided RWBS (VA-RWBS) technique is developed to solve a similar ML problem in a single-user communication system, where they iteratively detected the data sequences and estimated the channel coefficients. The scheme was referred to as joint channel estimation and data detection in [16]. More specifically, the signal detection-namely, the inner optimization - was carried out by using the standard VA, whereas the channel estimation - the outer optimization - was performed by the RWBS algorithm. However, the VA-RWBS scheme cannot directly be transplanted into the multiuser SDMA/OFDM systems considered in this paper because we should first separate the multiple users' data at the BS. Hence, the VA-RWBS algorithm cannot directly detect the users' signals in multiuser communication systems. To circumvent this problem, we propose the DRWBS-JCEMUD scheme, which is illustrated within the box surrounded by the dotted line in the right-hand side of Fig. 1. The proposed DRWBS-JCEMUD scheme alternately estimates the channel as well as the users' data and mutually exchanges these estimates between both populations to find the joint optimum. Furthermore, the DRWBSJCEMUD is capable of exploiting the beneficial convergence acceleration capability of an arbitrary FEC decoder, as seen in Fig. 1. More explicitly, the FEC decoder mitigates the effects of estimation errors, regardless of their source, and, hence, accelerates convergence and reduces the complexity by requiring fewer iterations.

In the following, we will use the respective CFs for estimating both the CIRs and the users' transmitted data. The reason for using two separate $\mathrm{CFs}$ - rather than one-is simply to reduce the complexity by decomposing the JCEMUD tasks into two simpler subtasks. The computational complexity will further be reduced when we carry out the MUD on a percarrier basis, whereas the accuracy of the channel estimator will be enhanced when we rely on all the data relevant for the estimated link. The pairs of CFs used in the RWBS-based channel estimator and MUD are formulated as

$$
\begin{aligned}
J_{\mathbf{h}}\left(\mathbf{h}_{p}[s] \mid \hat{\mathbf{X}}[s]\right) & =\left\|\mathbf{Y}_{p}[s]-\hat{\mathbf{X}}^{T}[s] \mathbf{F h}_{p}[s]\right\|^{2} \\
J_{\mathbf{X}}(\mathbf{X}[s, k] \mid \hat{\mathbf{H}}[s, k]) & =\|\mathbf{Y}[s, k]-\hat{\mathbf{H}}[s, k] \mathbf{X}[s, k]\|^{2}
\end{aligned}
$$

where the estimated signals of the block diagonal matrix $\hat{\mathbf{X}}[s] \in \mathbb{C}^{U K \times K}$ have a similar structure as $\mathbf{X}[s] \in \mathbb{C}^{U K \times K}$ in (5). Furthermore, the received data $\mathbf{Y}[s, k] \in \mathbb{C}^{P \times 1}$, the FDCHTFs $\hat{\mathbf{H}}[s, k] \in \mathbb{C}^{P \times U}$, and the users' transmitted data to be estimated, namely, $\mathbf{X}[s, k] \in \mathbb{C}^{U \times 1}$, are defined as

$$
\begin{aligned}
\mathbf{Y}[s, k] & =\left[Y_{1}[s, k], Y_{2}[s, k], \ldots, Y_{P}[s, k]\right]^{T} \\
\hat{\mathbf{H}}[s, k] & =\left[\begin{array}{ccc}
\hat{H}_{1}^{1}[s, k] & \ldots & \hat{H}_{1}^{U}[s, k] \\
\vdots & \ldots & \vdots \\
\hat{H}_{P}^{1}[s, k] & \ldots & \hat{H}_{P}^{U}[s, k]
\end{array}\right] \\
\mathbf{X}[s, k] & =\left[X^{1}[s, k], \ldots, X^{U}[s, k]\right]^{T} .
\end{aligned}
$$

The decision rule for the optimal JCEMUD is to find the specific channel coefficient matrix $\hat{\mathbf{h}}_{p}[s]$ and/or signal vector $\hat{\mathbf{X}}[s, k]$ that has the lowest cost (or highest fitness value). This is achieved with the aid of the RWBS-based global optimization technique.

\section{B. RWBS-Algorithm-Assisted Channel Estimation}

The RWBS [16] constitutes a guided random search-based global optimization algorithm. The basic philosophy of the RWBS algorithm is that by commencing from a search pool of the potential solutions, which is initially randomly populated, the algorithm continues by replacing the "lowest quality" solutions of the population with the "best" potential solutions generated by nature-inspired combinations/mutations of the candidate solutions in the pool until the process converges. The process is constituted by an amalgam of the mutation, evaluation, normalization, weighting, and construction steps, which will further be detailed later in this section. Specifically, we are interested in determining the CIR vector $\hat{\mathbf{h}}_{p}[s]$ and the data vector $\hat{\mathbf{X}}[s, k]$ that minimize the CFs of (9) and (10), 


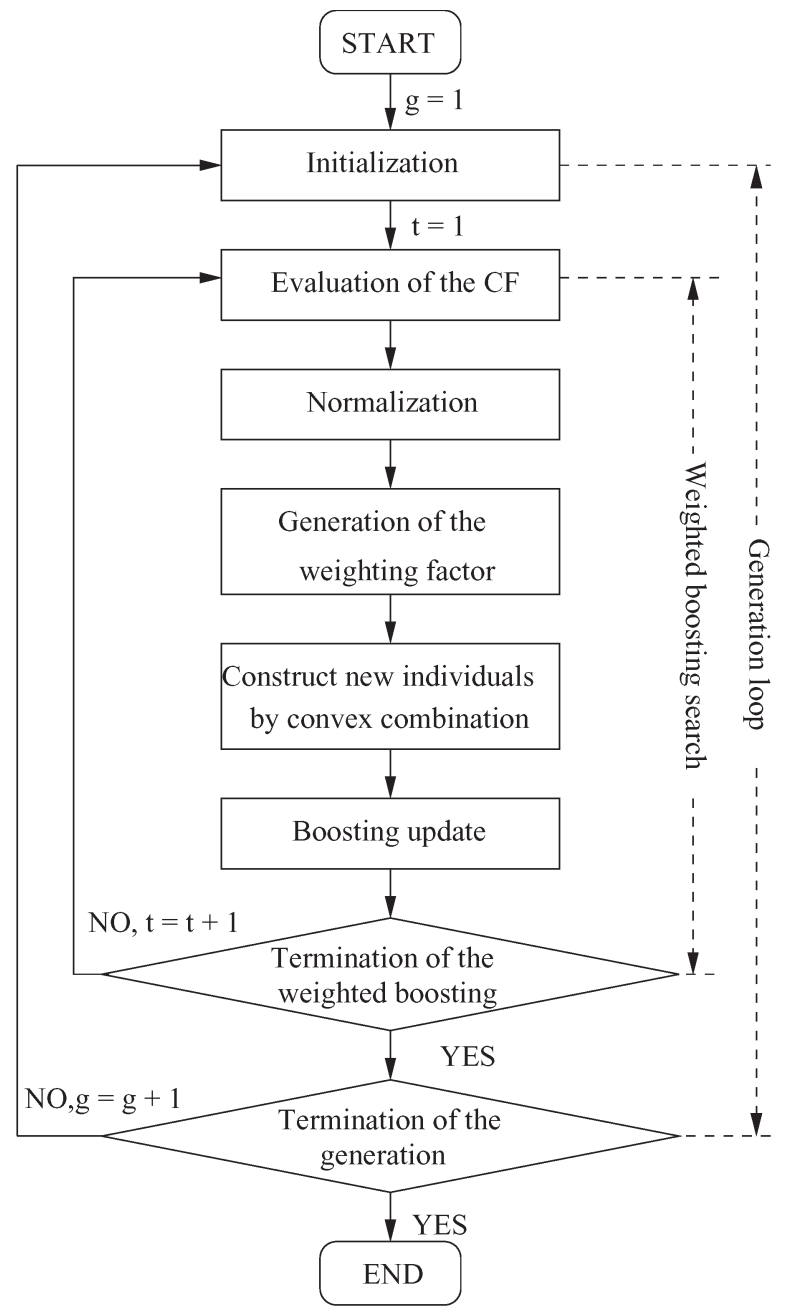

Fig. 2. Flowchart depicting the structure of the proposed RWBS algorithm used to jointly estimate the CIR coefficients and to detect the users' transmitted signals.

respectively. The principle of the proposed RWBS-based joint channel estimator and MUD can best be understood with the aid of the flowchart shown in Fig. 2, which will often be referred to during our forthcoming discourse. Subsequently, we limit our treatment to the channel estimator for reasons of space economy. ${ }^{1}$

1) Generation Initialization: Initialization of the RWBS is performed at every so-called generation for each optimization step, as seen in Fig. 2, by creating $P_{s}$ candidate solutions or individuals. The set of $P_{s}$ individuals is known as a population, where $P_{s}$ is the population size. These individuals represent the unknown variables of interest, which in this case are the CIR vector $\hat{\mathbf{h}}_{p}[s]$ in (9). We denote the complex-valued CIR coefficient individuals as $\hat{\mathbf{h}}_{p}^{\left(g, p_{s}\right)}[s]=$ $\left[\hat{\mathbf{h}}_{p}^{1,\left(g, p_{s}\right)}[s], \hat{\mathbf{h}}_{p}^{2,\left(g, p_{s}\right)}[s], \ldots, \hat{\mathbf{h}}_{p}^{U,\left(g, p_{s}\right)}[s]\right]^{T}$, where $g$ and $p_{s}$ represent the generation index and the index of an individual in the population, respectively.

More specifically, $P_{s}$ individuals are generated as $\hat{\mathbf{h}}_{p}^{(g, 1)}[s]=$ $\hat{\mathbf{h}}_{p, \text { best }}^{(g-1)}[s]$, where $\hat{\mathbf{h}}_{p, \text { best }}^{(g-1)}[s]$ is the best individual of generation

\footnotetext{
${ }^{1}$ The MUD's operation is similar to that of the channel estimator, with the slight difference that the MUD relies on the CF of (10).
}

$(g-1)$, which will be defined later in this section. The remaining $\left(P_{s}-1\right)$ individuals are then created by the mutation operator, which is the same as the GA's mutation operator [4], yielding

$$
\left\{\begin{aligned}
\mathfrak{R}\left(\hat{\mathbf{h}}_{p}^{\left(g, p_{s}\right)}[s]\right)= & \mathfrak{R}\left(\hat{\mathbf{h}}_{p}^{(g, 1)}[s]\right) \\
& +\mu \cdot \operatorname{randn}(U L, 1), p_{s}=2,3, \ldots, P s \\
\mathfrak{I}\left(\hat{\mathbf{h}}_{p}^{\left(g, p_{s}\right)}[s]\right)= & \mathfrak{I}\left(\hat{\mathbf{h}}_{p}^{(g, 1)}[s]\right) \\
& +\mu \cdot \operatorname{randn}(U L, 1), p_{s}=2,3, \ldots, P s
\end{aligned}\right.
$$

where $\operatorname{randn}(U L, 1)$ is a $(U L \times 1)$-element pseudorandom vector drawn from the normal distribution with zero mean and unit variance, whereas $\mu$ is known as the mutation parameter. $\mathfrak{R}$ and $\Im$ respectively denote the real and imaginary components of the specific CIRs $\hat{\mathbf{h}}_{p}^{\left(g, p_{s}\right)}[s]$ to be mutated.

2) Evaluation of the $C F$ : Associated with the $p_{s}$ th individual is a so-called $\mathrm{CF}$ value, which has to be evaluated, as seen in Fig. 2. The CF value, which is denoted by $J_{\mathbf{h}}^{\left(g, p_{s}\right)}$ for $p_{s}=1,2, \ldots, P_{s}$, is computed by substituting the elements of the $p_{s}$ th CIR coefficient individual $\hat{\mathbf{h}}_{p}^{\left(g, p_{s}\right)}[s]$ into the CF of (9). The users' transmitted data $\hat{\mathbf{X}}[s]$ used in this equation are given by the RWBS-based MUD. Before the RWBS-based MUD output data becomes available, we employ the pilot-symbolassisted CIR estimate. Here, we denote the best individual in this generation as $\hat{\mathbf{h}}_{p, \text { best }}^{(g)}[s]$, which has the minimal $\mathrm{CF}$ value

$$
\hat{\mathbf{h}}_{p, b e s t}^{(g)}[s]=\arg \min _{\hat{\mathbf{h}}_{p}^{\left(g, p_{s}\right)}[s]} J_{\mathbf{h}}\left(\mathbf{h}_{p}[s] \mid \hat{\mathbf{X}}[s]\right) .
$$

3) Normalization: To reflect the relative merit of an individual compared with other individuals of this generation, we normalize the $\mathrm{CF}$ value as follows:

$$
\bar{J}_{\mathbf{h}}^{\left(g, p_{s}\right)}=J_{\mathbf{h}}^{\left(g, p_{s}\right)} / \sum_{p_{s}=1}^{P_{s}} J_{\mathbf{h}}^{\left(g, p_{s}\right)}, \quad p_{s}=1,2, \ldots, P_{s} .
$$

The relative merit of the individual constitutes vital information, which will be used for generating the weighting factor guiding the random search toward the optimal solution.

4) Generation of the Weighting Factor: Intuitively, the meritorious individuals having low CF values should contribute toward generating new individuals. Hence, we introduce weighting factors for reflecting the relative merits of the corresponding individuals, which will be perpetually modified according to their normalized $\mathrm{CF}$ values as follows:

1) Compute the weighting factor $\beta_{h}^{t}$ according to

$$
\beta_{h}^{t}=\frac{\eta_{h}^{t}}{1-\eta_{h}^{t}}, \eta_{h}^{t}=\sum_{p_{s}=1}^{P_{s}} \delta_{h}^{\left(g, p_{s}\right)}(t-1) \bar{J}_{\mathbf{h}}^{\left(g, p_{s}\right)}
$$

where $\delta_{h}^{\left(g, p_{s}\right)}(t-1)$ are the weights of the individuals representing the CIR coefficients of $\hat{\mathbf{h}}_{p}^{\left(g, p_{s}\right)}[s]$, whereas $t$ represents the iteration index in the weighted boosting aided search. The weighting factor $\beta_{h}^{t}$ used for updating the weights $\delta_{h}^{\left(g, p_{s}\right)}(t)$ is not fixed, it rather changes at each iteration of the weighted boosting-aided search according 
to $\eta_{h}^{t}$. The updated weighting factor $\beta_{h}^{t}$ decreases with the reduction of $\eta_{h}^{t}$, which in turn increases the difference between $\delta_{h}^{\left(g, p_{s}\right)}(t)$ and $\delta_{h}^{\left(g, p_{s}\right)}(t-1)$.

2) Update the weights as

$$
\delta_{h}^{\left(g, p_{s}\right)}(t)= \begin{cases}\delta_{h}^{\left(g, p_{s}\right)}(t-1)\left(\beta_{h}^{t}\right)^{\bar{J}_{\mathbf{h}}^{\left(g, p_{s}\right)},}, & \beta_{h}^{t} \leq 1 \\ \delta_{h}^{\left(g, p_{s}\right)}(t-1)\left(\beta_{h}^{t}\right)^{1-\bar{J}_{\mathbf{h}}^{\left(g, p_{s}\right)},}, & \beta_{h}^{t}>1\end{cases}
$$

and then normalize them as follows:

$$
\delta_{h}^{\left(g, p_{s}\right)}(t)=\frac{\delta_{h}^{\left(g, p_{s}\right)}(t)}{\sum_{p_{s}=1}^{P_{s}} \delta_{h}^{\left(g, p_{s}\right)}(t)}, \quad p_{s}=1,2, \ldots, P_{s} .
$$

The weights $\delta_{h}^{\left(g, p_{s}\right)}(t-1)$ reflect the fitness ratios of the individuals, which are used for constructing new individuals for the next generation. All of the weights $\delta_{h}^{\left(g, p_{s}\right)}(t-1)$ assigned throughout all the iterations $t=$ $1,2, \cdots$ will be nonnegative and sum to one, yielding $\sum_{p_{s}=1}^{P_{s}} \delta_{h}^{\left(g, p_{s}\right)}(t-1)=1$. Naturally, if we have no reason to favor any of the individuals, we can set all of the initial weights to $\delta_{h}^{\left(g, p_{s}\right)}(0)=1 / P_{s}$. The reason for differently updating the weights for the two scenarios according to $\beta_{h}^{t} \lesseqgtr 1$ is that the exponential function of $\left(\beta_{h}^{t}\right)^{x}$ is monotonically increasing when we have $\beta_{h}^{t}>$ 1 , and it is monotonically decreasing for $\beta_{h}^{t}<1$. To augment the influence of meritorious individuals (having low CF values) and to deweight the deficient individuals (having high $\mathrm{CF}$ values), the weights are updated using the multiplicative rule of (18).

5) Convex Combination: Convex combination is a linear combination of the given vectors, which is employed to explore all the possible combinations within the convex hull ${ }^{2}$ of the given vectors by updating the weights in the boosting search, as seen in Fig. 2. More formally, given a finite number of individuals representing the CIR coefficients, a convex combination of these individuals is created to construct a new individual in the form of

$$
\hat{\mathbf{h}}_{p}^{\left(g, P_{s}+1\right)}[s]=\sum_{p_{s}=1}^{P_{s}} \delta_{h}^{\left(g, p_{s}\right)}(t) \hat{\mathbf{h}}_{p}^{\left(g, p_{s}\right)}[s] .
$$

Intuitively, we can see that the convex combination of (20) is capable of exploiting the meritorious individuals of low $\mathrm{CF}$ values (having high weights) to produce individuals that may have an even lower CF value. A "mirror image" of $\hat{\mathbf{h}}_{p}^{\left(g, P_{s}+1\right)}[s]$ is also considered as a new individual, which is generated with respect to $\hat{\mathbf{h}}_{p, \text { best }}^{(g)}[s]$ and along the direction defined by $\left(\hat{\mathbf{h}}_{p, \text { best }}^{(g)}[s]-\hat{\mathbf{h}}_{p}^{\left(g, P_{s}+1\right)}[s]\right)$ as follows:

$$
\hat{\mathbf{h}}_{p}^{\left(g, P_{s}+2\right)}[s]=\hat{\mathbf{h}}_{p, \text { best }}^{(g)}[s]+\left(\hat{\mathbf{h}}_{p, \text { best }}^{(g)}[s]-\hat{\mathbf{h}}_{p}^{\left(g, P_{s}+1\right)}[s]\right) .
$$

\footnotetext{
${ }^{2}$ Convex hull is the minimal convex set containing all the given vectors [15]. The vectors refer to the individuals in this paper.
}

6) Weighted Boosting Update: The two new individuals generated by convex combination will compete for superseding the worst individual according to their $\mathrm{CF}$ values. The better individual of $\hat{\mathbf{h}}_{p}^{\left(g, P_{s}+1\right)}[s]$ and $\hat{\mathbf{h}}_{p}^{\left(g, P_{s}+2\right)}[s]$ then substitutes the worst individual $\hat{\mathbf{h}}_{p \text {,worst }}^{(g)}[s]$ and becomes part of the next iteration of the boosting search. Here, the worst individual $\hat{\mathbf{h}}_{p, \text { worst }}^{(g)}[s]$ is defined as

$$
\hat{\mathbf{h}}_{p, \text { worst }}^{(g)}[s]=\arg \max _{\hat{\mathbf{h}}_{p}^{\left(g, p_{s}\right)}[s]} J_{\mathbf{h}}\left(\mathbf{h}_{p}[s] \mid \hat{\mathbf{X}}[s]\right) .
$$

7) Termination: There are two termination criteria: 1) judge whether to terminate the weighted boosting search, and 2 judge whether to terminate the generation search. We will elaborate upon both in the following.

1) Determine whether to terminate the weighted boosting search. If we have $\left\|\hat{\mathbf{h}}_{p}^{\left(g, P_{s}+1\right)}[s]-\hat{\mathbf{h}}_{p}^{\left(g, P_{s}+2\right)}[s]\right\|<\xi_{\mathbf{h}}$, i.e., there is no further substantial improvement in the boosting search, then we will terminate the boosting search of this generation and go to the next step in Fig. 2 to determine whether to terminate the generation search. Here, $\xi_{\mathbf{h}}$ represents the accuracy that has to be reached before terminating the weighted boosting search.

2) Determine whether to proceed to the next generation. If we have $\left\|\hat{\mathbf{h}}_{p, \text { best }}^{(g)}[s]-\hat{\mathbf{h}}_{p, \text { best }}^{(g-1)}[s]\right\|<\zeta_{\mathbf{h}}$ and $g>\tilde{G}$, then curtail the search in Fig. 2, and use the final solutions: $\hat{\mathbf{h}}_{p}[s]=\hat{\mathbf{h}}_{p, \text { best }}^{(g)}[s]$, or go to the generation initialization step. Here, $\zeta_{\mathbf{h}}$ quantifies the accuracy that has to be satisfied, whereas $\tilde{G}$ is the minimal number of repeated generation search loops to be visited. The reason for this setting is to guarantee that a sufficiently high number of repeats or generations have been considered to find the globally optimal solution.

Again, it is worth pointing out that the proposed DRWBSJCEMUD conveniently generates the soft bit values or LLRs associated with the $m_{B}$ th bit position of the $u$ th user's transmitted $M$-QAM-modulated signal $\hat{\mathbf{X}}[s, k]$ upon invoking the maximum approximation technique of [3], which yields ${ }^{3}$

$$
\begin{aligned}
\mathcal{L}_{u, m_{B}} \approx-\frac{1}{\sigma_{n}^{2}} & {\left[\left\|\mathbf{Y}[s, k]-\hat{\mathbf{H}}[s, k] \hat{\mathbf{X}}_{u, m_{B}, 0}[s, k]\right\|^{2}\right.} \\
& \left.-\left\|\mathbf{Y}[s, k]-\hat{\mathbf{H}}[s, k] \hat{\mathbf{X}}_{u, m_{B}, 1}[s, k]\right\|^{2}\right]
\end{aligned}
$$

where the FD-CHTF $\hat{\mathbf{H}}[s, k]$ may readily be obtained after we acquire the CIR coefficient estimate $\hat{\mathbf{h}}_{p}[s]$. The notation of $\hat{\mathbf{X}}_{u, m_{B}, b}[s, k], b=0,1$ suggests that the $m_{B}$ th bit position of the $u$ th user's signal on the $k$ th subcarrier of the $s$ th OFDM symbol is $b$, whereas the other users' signals are the same as those in $\hat{\mathbf{X}}[s, k]$. However, as to the $M$-QAM scheme, there are $M / 2$ signals, whose $m_{B}$ th bit position of the $u$ th user's

\footnotetext{
${ }^{3}$ Note that normalization was applied for $\mathcal{L}_{u, m_{B}}$ as that in [4, p. 238] $\tilde{\mathcal{L}}_{u, m_{B}}=\min \left\{\mathcal{L}_{u, m_{B}}, P \times U\right\}$, where $P \times U$ represents the normalization
} factor. 
signal is 0 or 1 . We select the most likely signal based on the following criterion given in (24), shown at the bottom of the page, where $\mathcal{M}_{u, m_{B}, b}^{U}$ denotes the specific constellation subset associated with the $u$ th user. More explicitly, $\mathcal{M}_{u, m_{B}, b}^{U}$ is constituted by those specific trial vectors, whose $u$ th element's $m_{B}$ th bit has a value of $b$, which is expressed as (25), shown at the bottom of the page, where $\mathcal{M}_{C}$ denotes the constellation set containing the $2^{m}$ legitimate complex-valued constellation points associated with the specific $M$-QAM scheme employed, and " $\cap$ " is the intersection operation. Explicitly, (25) suggests that the $\left(m_{B}\right)$ th bit of $\hat{X}^{u}[s, k]$ has a value of $b$. Still referring to (25), $m$ denotes the number of bits per $M$-QAM symbol.

\section{Discussions on Computational COMPLEXITY AND CONVERGENCE}

\section{A. Computational Complexity}

The computational complexity of the proposed scheme is predominantly determined by the population size $P_{s}$, by the number of generations $\tilde{G}$ required to approach convergence, and by the number of boosting search steps $T_{\mathrm{bs}}$ at each generation. Given the population size $P_{s}$ and a fixed number of $T_{\mathrm{bs}}$ boosting search steps at each generation of the RWBS algorithm, the number of $\mathrm{CF}$ evaluations (CF-Evals.) required at each generation is equal to $\left[\left(P_{s}-1\right)+2 T_{\mathrm{bs}}\right]$, where $\left(P_{s}-\right.$ $1)$ is the number of CF-Evals. outside the boosting search, and $2 T_{\mathrm{bs}}$ is the number of CF-Evals. in the boosting search. Hence, the total CF-Evals. required to detect the users' transmitted signals at each subcarrier of each OFDM symbol is equal to $\left[\left(P_{s}-1\right)+2 T_{\mathrm{bs}}\right] \times \tilde{G}$. We denote the computational complexity of the RWBS-based MUD as $O\left(\left[(P s-1)+2 T_{\mathrm{bs}}\right] \times \tilde{G}\right)$. We can see that the computational complexity of the proposed DRWBS-JCEMUD scheme is independent of the order $M$ of $M$-QAM and the number of users $U$. To explicitly quantify the complexity of the RWBS-based optimization algorithm, we compare it with that of the MMSE-MUD and the optimum ML-MUD. The computational complexity of the MMSE-MUD is dominated by the matrix inversion of a $(U \times U)$-element matrix; hence, its computational complexity can be approximated as $O\left(U^{3}\right)$. The computational complexity of the ML-MUD using exhaustive search is equivalent to $M^{U}$ for a $U$-user SDMA/OFDM system employing $M$-QAM, i.e., $O\left(M^{U}\right)$. We will show that the DRWBS-JCEMUD scheme is capable of attaining near-optimum performance at a lower computational complexity than the optimum ML-MUD, particularly for high-order $M$-QAM scenarios.
It is worth pointing out that the proposed RWBS generating the aforementioned soft outputs in the form of LLRs for multiple users imposes only a modest complexity increase in comparison with the single-user ML detection scenario. This is because the search space required for finding the best potential signal of user- $u$ according to (24) is the same as that of a singleuser scenario, which is an explicit benefit of employing the maximum approximation in [3]. The resultant output LLRs can then be directly fed to the channel decoder in Fig. 1 to improve the multiuser MIMO SDMA/OFDM system's performance.

\section{B. Convergence}

Similar to any other stochastic optimization algorithm, such as GAs and evolutionary algorithms, the convergence of the RWBS algorithm is characterized by its probability of convergence, which is defined as [17]

$$
\lim _{g \rightarrow+\infty} \operatorname{Pr}\left(\left|\hat{\mathbf{h}}_{p, \text { best }}^{(g)}[s]-\mathbf{h}_{p}[s]\right|>\varepsilon\right)=0
$$

where $\operatorname{Pr}(\cdot)$ represents the probability of the event considered, and $\varepsilon$ is a small positive value. Equation (26) suggests that the solutions are located outside the $\varepsilon$-neighborhood of $\mathbf{h}_{p}[s]$ with a probability of zero as the RWBS proceeds.

There exists a probability $p_{g}>0$ at each generation that the individuals in the parental populations generate an offspring $\hat{\mathbf{h}}_{p, \text { best }}^{(g)}[s]$ belonging to the $\varepsilon$ neighborhood of $\mathbf{h}_{p}[s]$. These $p_{g}$, $g=1,2, \cdots$ values may be different at different generations. As a benefit of the elitism, the best individuals of the next generation are at least as good as or better than its counterpart in the current generation, which indicates that the probability $p_{g}$ is monotonically increasing for $g=1,2, \cdots$. Hence, this feature will lead to the following proposition:

$$
\lim _{g \rightarrow+\infty} \operatorname{Pr}\left(\left|\hat{\mathbf{h}}_{p, b e s t}^{(g)}[s]-\mathbf{h}_{p}[s]\right|<\varepsilon\right)=1
$$

which indicates that the populations will convergence to the $\varepsilon$ neighborhood of $\mathbf{h}_{p}[s]$ with a unity probability as the RWBS proceeds.

\section{Simulation Results ANd Discussions}

In this section, we will investigate the achievable performance of the multiuser MIMO SDMA/OFDM system using the proposed DRWBS-JCEMUD scheme. As an example, a simple four-path Rayleigh fading channel model is employed

$$
\hat{\mathbf{X}}_{u, m_{B}, b}[s, k]=\arg \left\{\min _{\hat{\mathbf{X}}[s, k] \in \mathcal{M}_{u, m_{B}, b}^{U}}\|\mathbf{Y}[s, k]-\hat{\mathbf{H}}[s, k] \hat{\mathbf{X}}[s, k]\|^{2}\right\}, b=0,1
$$

$$
\mathcal{M}_{u, m_{B}, b}^{U}=\left\{\hat{\mathbf{X}}[s, k]=\left[\hat{X}^{1}[s, k], \hat{X}^{2}[s, k], \ldots, \hat{X}^{U}[s, k]\right]^{T} \mid\left\{\hat{X}^{u}[s, k] \in \mathcal{M}_{C}\right\} \cap\left\{b_{u, m_{B}}=b\right\}\right\}
$$


TABLE I

Basic Simulation Parameters USED IN OUR Simulations

\begin{tabular}{||l|l|l||}
\hline \hline \multirow{5}{*}{ LDPC } & Modulation scheme & 16 -QAM \\
& Code rate & $1 / 2$ \\
& Maximum iterations & 50 \\
& Codeword length & 1152 \\
\hline \multirow{5}{*}{ Channel } & No. of paths $L$ & 4 \\
& Delay & $0,1, \cdots, 3$ \\
& Average path gains & {$[0 ;-5 ;-10 ;-15](d B)$} \\
& $F_{D}$ & 0.003 \\
& Doppler spectrum & $\mathrm{U}$-shaped \\
& No. of the MSs $U$ & 4 \\
& No. of the received antennas $P$ & 4 \\
& Subcarriers $K$ & 64 \\
& Cyclic prefix $K_{c p}$ & 16 \\
\hline \multirow{5}{*}{ DRWBS- } & Initialization population & Randomly generate \\
& Pilot overhead $\epsilon$ & $6.25 \%$ \\
Population size $P_{s}$ & 40 (unless specified) \\
& Mutation parameter $\mu$ & 0.01 (unless specified) \\
Initialization weights $\delta^{\left(g, p_{s}\right)}(0)$ & $1 / P_{s}$ \\
& Accuracy to be reached of & \\
& the weighted boosting search $\xi$ & $10^{-5}$ (unless specified) \\
& Accuracy to be reached of \\
& the generations search $\zeta$ & $10^{-5}$ (unless specified) \\
\hline \hline
\end{tabular}

for each transmit-antenna-receive-antenna link, and each path has a U-shaped Doppler spectrum for its diffuse component. Each of the paths experiences independent Rayleigh fading having the same Doppler frequency of $F_{D}=f_{d} T_{s}$ normalized to the OFDM symbol rate, where $f_{d}$ and $T_{s}$ are the maximum Doppler frequency and the OFDM symbol duration including the $\mathrm{CP}$. The average path gains are $[0 ;-5 ;-10 ;-15] \mathrm{dB}$, whereas the delays of each path are $0,1,2$ and 3 , which have been normalized to the sample duration of the input signals. Moreover, a half-rate $r=1 / 2$ binary low density parity check (LDPC) code having a block length of $L_{L D P C}=1152$ is employed. The modulation scheme is 16-QAM for all users. The BS has an array of $P=4 \mathrm{UL}$ receiver antennas while supporting $U=4$ UL MSs simultaneously transmitting their data. The algorithmic parameters of the DRWBS-JCEMUD scheme used in our simulations are summarized in Table I.

\section{A. Channel Estimation Performance}

Fig. 3 shows the evolution of the mean square error (MSE) of the estimated CIRs versus the generation index for different values of $E_{b} / N_{0}$, respectively. We can see from Fig. 3 that the MSE is reduced upon increasing the generation index, and it approaches the noise floor.

As the DRWBS-JCEMUD scheme is an iteration-based algorithm, the number of generations used is an important parameter directly determining the complexity of the proposed DRWBSJCEMUD scheme. In Fig. 4, we portray the average number of CF-Evals. that are required to approach convergence versus the population sizes $P_{s}$ and mutation parameters $\mu$. It can be seen from Fig. 4 that the number of generations required decreases upon increasing the population size at a certain fixed mutation parameter. With regard to the effect of the mutation parameter $\mu$, we can see from Fig. 4 that a larger mutation parameter typically necessitates more CF-Evals., but by contrast, the number of CF-Evals. required for convergence slowly increases when $\mu<0.04$. By contrast, it steeply increases for $\mu>0.04$, and there is a ridge at $P_{s}=80$ in Fig. 4.

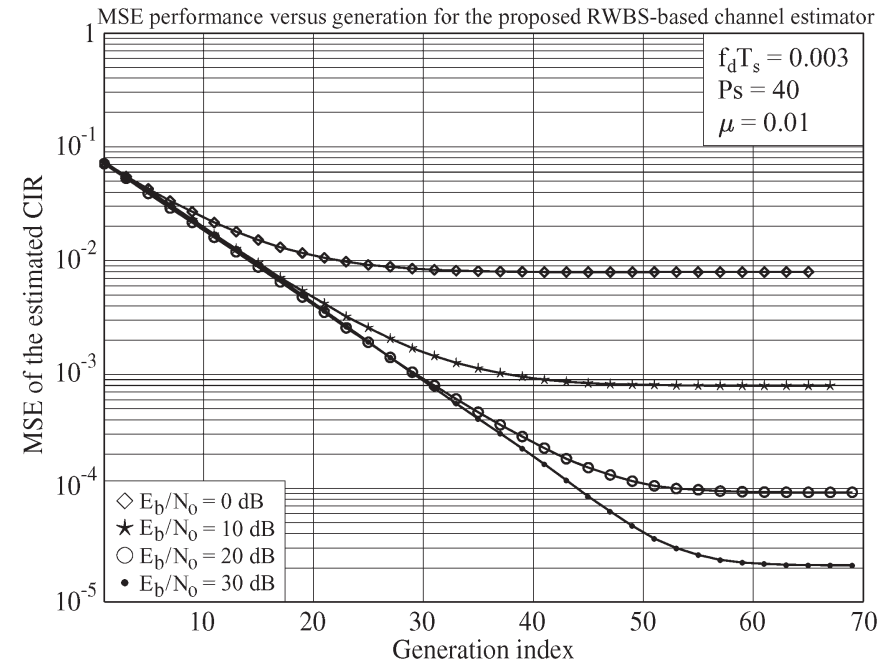

Fig. 3. MSE performance against the repeated generations required for convergence of the proposed DRWBS-JCEMUD scheme.

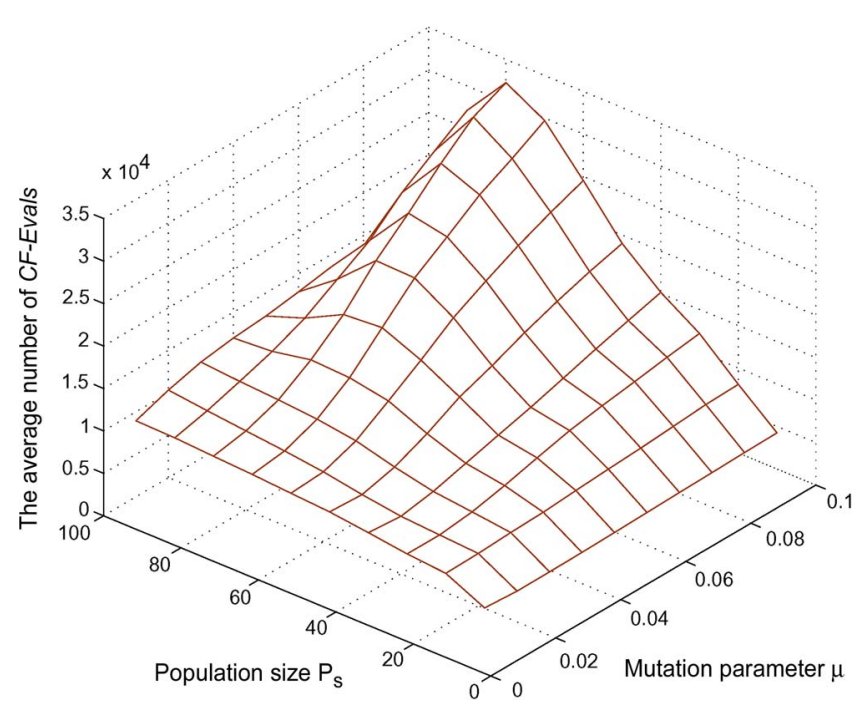

Fig. 4. Average number of generations required for the convergence of the proposed DRWBS-JCEMUD scheme versus the population size $P_{s}$ and the mutation parameter $\mu$ at $E_{b} / N_{0}=20 \mathrm{~dB}$.

To provide further insights, in Fig. 5, we portray the histogram of the number of generations required for approaching convergence at $E_{b} / N_{0}=20 \mathrm{~dB}$. The parameters used in these investigations are given in the caption of the figure. These results were obtained from the statistical evaluation of 288 independent CIR estimation events based on the RWBS algorithm. The $x$-axis represents the number of generations required for the RWBS-based CIR estimator to converge, whereas the "Ratio" on the $y$-axis shows what proportion of the independent CIR estimation events required the particular number of generations shown on the $x$-axis. As an example, about $11 \%$ or 32 independent CIR estimation events required 69 generations for the RWBS algorithm to acquire the converged estimate of the CIR, which is represented by the height of the bar seen in Fig. 5.

Fig. 6 examines the effects of different population sizes $P_{s}$ and mutation parameters $\mu$ on the achievable MSE at $E_{b} / N_{0}=$ $20 \mathrm{~dB}$. Observe in Fig. 6 that the population size has an insignif- 


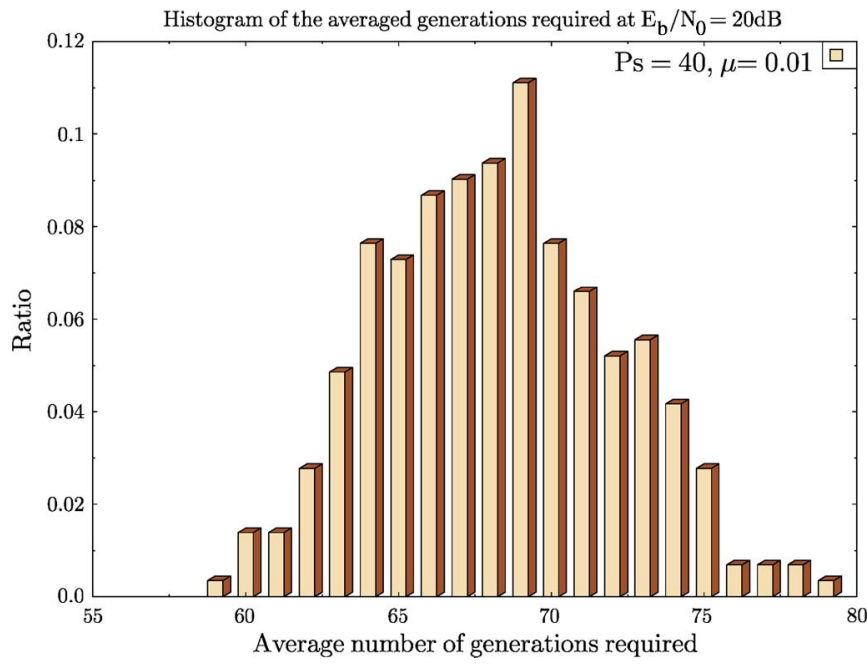

Fig. 5. Histogram of the number of generations required for the proposed DRWBS-JCEMUD scheme to converge at $E_{b} / N_{0}=20 \mathrm{~dB}$, where $f_{d} T_{s}=$ $0.003, P_{s}=40$, and $\mu=0.01$.

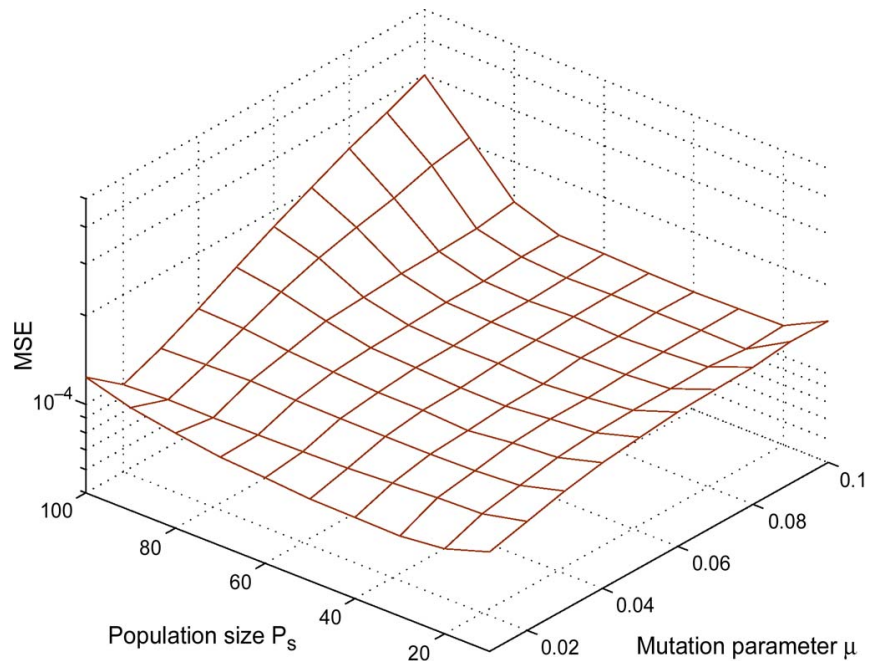

Fig. 6. Achievable MSE performance versus population size $P_{s}$ and mutation parameter $\mu$ at $E_{b} / N_{0}=20 \mathrm{~dB}$.

icant impact on the achievable MSE when $\mu<0.04$. However, ironically, larger population sizes perform worse than smaller population sizes for $\mu>0.04$. As regards to the mutation parameter, smaller mutation parameters typically outperform the larger parameters.

\section{B. Bit Error Rate (BER) Performance of the RWBS-Based MUD}

In Fig. 7, we characterize the BER versus complexity behavior of the proposed solution, where the complexity is increased by increasing the number of generations. We set $E_{b} / N_{0}=10$ and $20 \mathrm{~dB}$ and consider population sizes of $P_{s}=20,40,60$, and 80 . The lower bound of the achievable BER is given by the optimal ML-MUD. Note that for $E_{b} / N_{0}=20 \mathrm{~dB}$, the optimal ML-MUD attains an infinitesimally low BER. It can be seen from Fig. 7 that the proposed schemes exhibit a performance gap to the lower-bound BER of the optimal ML-MUD at $E_{b} / N_{0}=10 \mathrm{~dB}$ due to the residual multiuser interference and the high-level noise. By contrast, they converge rapidly at $E_{b} / N_{0}=20 \mathrm{~dB}$, hence accurately detecting the transmitted

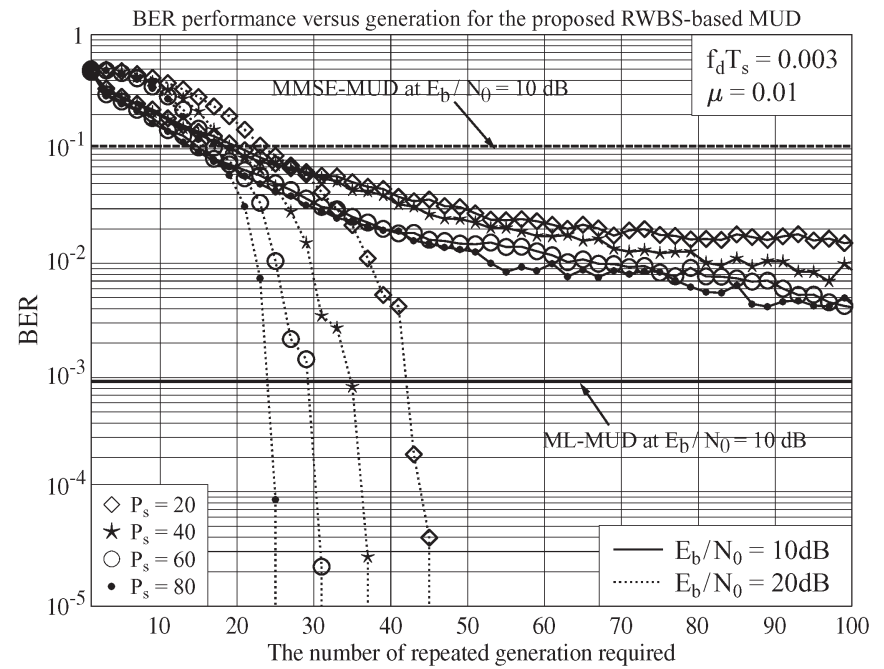

Fig. 7. BER performance against the number of generations required for the proposed DRWBS-JCEMUD scheme, assuming that 16-QAM modulation and half-rate LDPC channel codes of 1152 is employed by all $U=4$ users.

signals. Furthermore, the proposed RWBS-based MUD requires less generations at larger population sizes compared with its counterpart associated with smaller population sizes, which indicates that the RWBS algorithm has a higher "convergence speed."

Monte Carlo simulations of 100 independent runs were carried out to examine the stability of the proposed DRWBSJCEMUD scheme for a set of fixed but differently apportioned computational complexities of $\left[(P s-1)+2 T_{\mathrm{bs}}\right] \times$ $G=8000$, where we set $T_{\mathrm{sb}}=20$ in our simulations. Observe from Fig. 8 that the system's BER spreads across the range of $9.0 \times 10^{-4}$ and $5.0 \times 10^{-3}$. Naturally, the highest probability BER values represented by the highest bars in Fig. 8 dominate the overall performance. Furthermore, it can be seen that the proposed DRWBS-JCEMUD scheme exhibits the lowest bar spread for the parameter combinations of $P s=40, G=100$ and $P s=50, G=90$, which allows us to infer the loose relationship of $\left(P s+2 T_{\mathrm{bs}}\right) \approx G$. In the following section, we will investigate the overall performance of the proposed DRWBSJCEMUD scheme.

\section{JCEMUD Performance}

In Fig. 9, the average MSE of the CIR estimation achieved by the proposed DRWBS-JCEMUD scheme at different number of iterations is evaluated. The "include FEC" scenario exploited the error correction capability of the LDPC code of Table I during each iteration, as seen in Fig. 1. By contrast, "exclude FEC" represents the case when the data output by the RWBSbased MUD is directly fed back to the RWBS-based CIR estimator without the LDPC code's assistance. Observe from Fig. 9 that the MSE performance of the CIR estimator is beneficially improved at Iter $=1^{4}$ when we exploit the error

\footnotetext{
${ }^{4}$ More specifically, Iter $=1$ implies that the classic decision-directed process is invoked for feeding back all the data output by the FEC-decoder to the RWBS-based channel estimator, where all the received data are used as pilot symbols.
} 


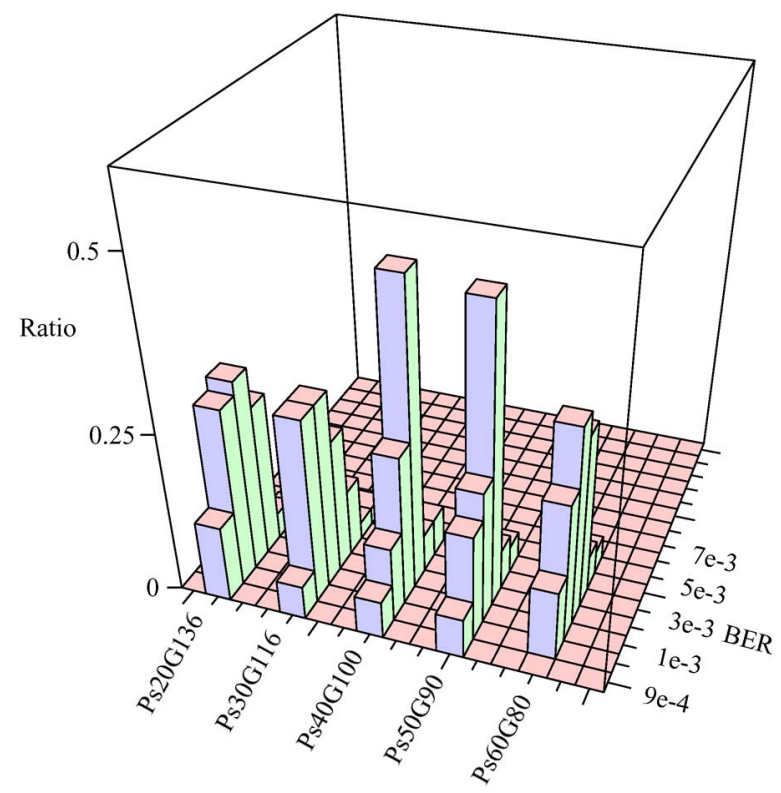

Fig. 8. Histogram of the BER for the proposed DRWBS-JCEMUD scheme, where $f_{d} T_{s}=0.003$ and $E_{b} / N_{0}=12 \mathrm{~dB}$. The number of boosting search is set as $T_{\mathrm{sb}}=20$ in our simulations. The quantity "Ratio" on the $z$-axis represents the proportion of experiments having a certain value of the system's BER from a total of 100 independent Monte Carlo simulations.

correction capability of the FEC decoder, approaching that of the CRLB benchmarker of [18], which is given by

$$
\operatorname{CRLB}(\mathbf{h})=\frac{\sigma_{n}^{2}}{K E_{s}}
$$

where $\sigma_{n}^{2}$ represents the noise variance, and $E_{s}$ is the average power of the transmitted signals. Note that the CRLB in (28) has been normalized to the number of paths. However, if we do not exploit the error correction capability of the LDPC decoder, then the estimator may perform even worse than the initial estimate, which may be due to error propagation. Similar to the average MSE performance trends characterized in Fig. 9, the systems's BER performance seen in Fig. 10 is also beneficially improved at Iter $=1$, when we include the LDPC decoder within the iterations.

To provide an overall impression of the attainable system performance, we evaluate the system's BER performance in Fig. 11 for a set of fixed but differently apportioned computational complexities of $\left[(P s-1)+2 T_{\mathrm{bs}}\right] \times G=8000$, where $T_{\mathrm{sb}}=20$ in our simulations. The conventional MMSE-MUD [3] and the optimal ML-MUD exploiting perfect CIRs are also included as references. Observe from Fig. 11 that a beneficial iteration gain is achieved by exploiting the error correction capability of the LDPC decoder to iteratively exchange information between the RWBS-based detector and the RWBS-based channel estimator. This reduces the performance discrepancy with respect to the ML-MUD relying on perfect CIRs to less than $1 \mathrm{~dB}$ at Iter $=1$ at a total computational complexity of $(2 \times$ $8000)=16,000$. Furthermore, the proposed scheme reduces the computational complexity to $(1-($ Iter $\times[(P s-1)+$ $\left.\left.\left.2 T_{\mathrm{bs}}\right] \times G / M^{U}\right)\right)=\left(1-\left(16000 / 16^{4}\right)\right) \approx 0.756$ at the cost of a 1-dB performance penalty, in comparison with the ML-MUD for $E_{b} / N_{0}>9 \mathrm{~dB}$. More specifically, the BER performance of

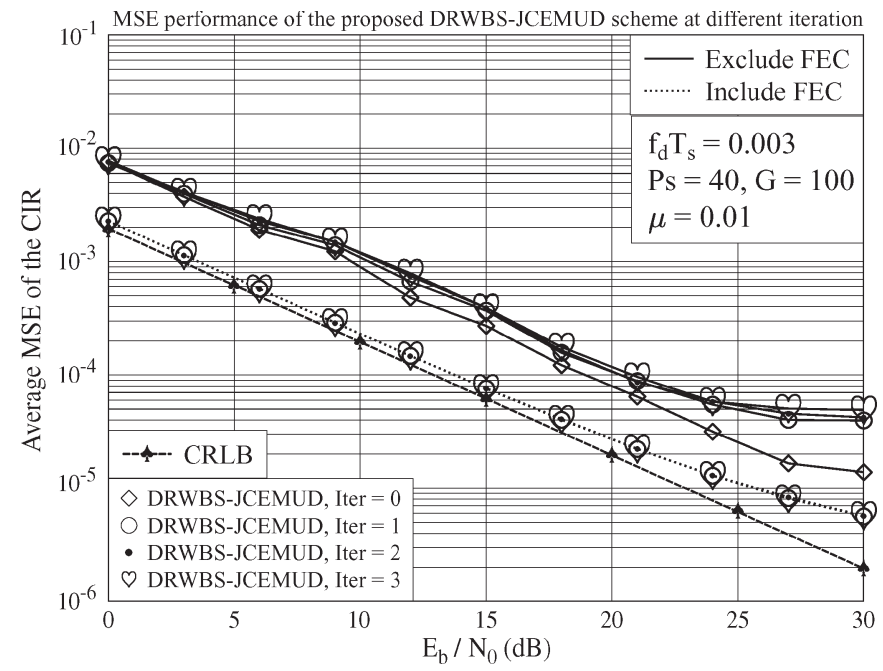

Fig. 9. MSE performance for the proposed DRWBS-JCEMUD scheme, which employs $6.25 \%$ pilots at Iter $=0$. After Iter $=0$, decision-directed channel estimation is used, implying that all of the data output by the RWBSbased MUD are used as pilots.

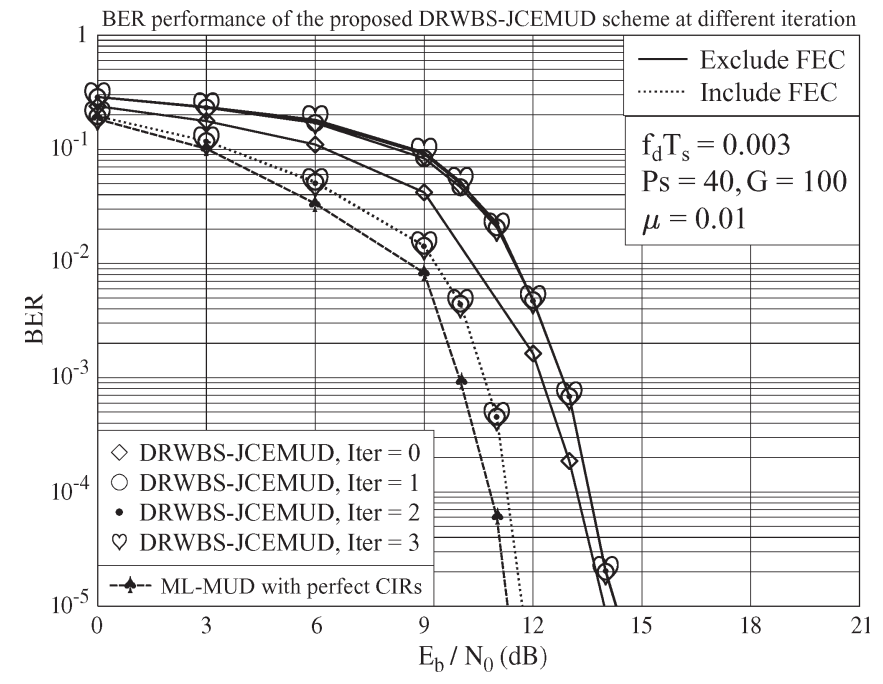

Fig. 10. BER performance for the proposed DRWBS-JCEMUD scheme, which employs $6.25 \%$ pilots at Iter $=0$. After Iter $=0$, decision-directed channel estimation is used, implying that all of the data output by the RWBSbased MUD are used as pilots. To be fair, the BER performances of the "exclude FEC" scenario are also recorded after FEC decoder for each iteration, although the FEC decoder does not participate into the decision direct iteration.

the proposed DRWBS-JCEMUD scheme remains by and large the same for all of the configurations considered at a fixed computational complexity for $E_{b} / N_{0}$ values below $6 \mathrm{~dB}$ and above $11 \mathrm{~dB}$. By contrast, a moderate population size combined with a moderate number of generations, which is given by $\left(P s+2 T_{\mathrm{bs}}\right) \approx G$ for the parameter combinations considered, is found to be more beneficial for $6 \mathrm{~dB}<E_{b} / N_{0}<11 \mathrm{~dB}$.

\section{CONCLUSION}

In this paper, we have proposed a random guided search scheme for JCEMUD, which was investigated in the context of MIMO SDMA/OFDM systems. The DRWBS-JCEMUD scheme is capable of approaching the performance of the optimal ML-MUD in conjunction with the randomly generated 


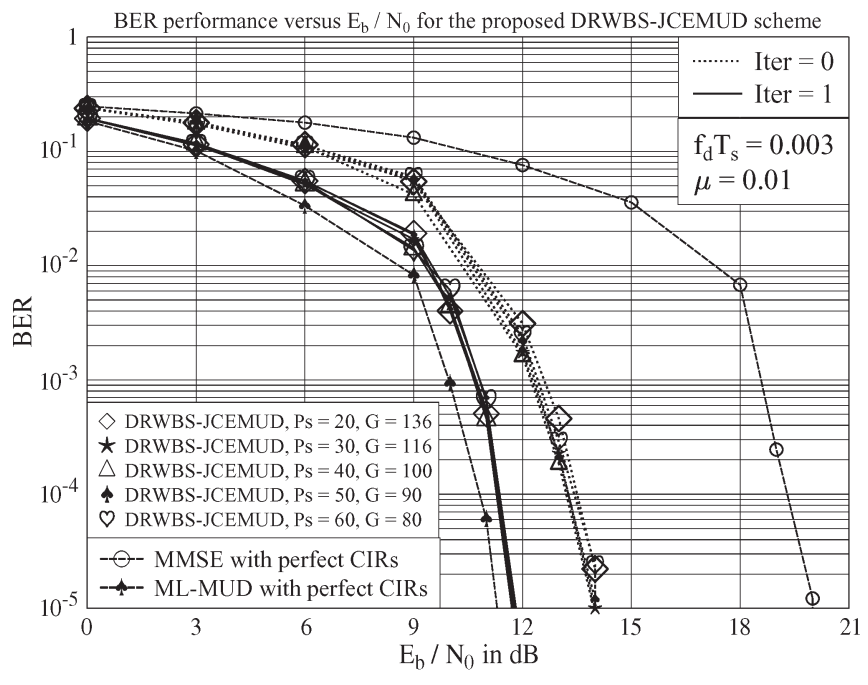

Fig. 11. BER versus $E_{b} / N_{0}$ performance of the LDPC-coded multiuser MIMO OFDM/SDMA system. The system supports four MSs simultaneously transmitting data to the BS, where the BS employs four antennas. The conventional MMSE and the optimal ML MUD with perfect channel knowledge are also given as references.

initial values. The effects of the algorithmic parameters of the DRWBS-JCEMUD scheme were explored using Monte Carlo simulations. The DRWBS-JCEMUD scheme advocated was capable of generating soft outputs for the channel decoder. As an example, at $E_{b} / N_{0}=10 \mathrm{~dB}$, a complexity reduction factor of 0.756 was achieved at the cost of a $1-\mathrm{dB}$ performance penalty in comparison with the ML-MUD.

\section{REFERENCES}

[1] J. Gao and H. Liu, "Low-complexity MAP channel estimation for mobile MIMO-OFDM systems," IEEE Trans. Wireless Commun., vol. 7, no. 3, pp. 774-780, Mar. 2008.

[2] P. Vandenameele, L. Van Der Perre, M. Engels, B. Gyselinckx, and H. De Man, "A combined OFDM/SDMA approach," IEEE J. Sel. Areas Commun., vol. 18, no. 11, pp. 2312-2321, Nov. 2000.

[3] L. Hanzo, M. Münster, B. J. Choi, and T. Keller, OFDM and MC-CDMA for Broadband Multi-User Communications, WLANs, and Broadcasting. Piscataway, NJ: IEEE Press, 2003.

[4] L. Hanzo, Y. Akhtman, L. Wang, and M. Jiang, MIMO-OFDM for LTE, WIFI and WIMAX: Coherent Versus Non-Coherent and Cooperative Turbo-Transceivers. Hoboken, NJ: Wiley, 2010.

[5] M. Jiang, J. Akhtman, and L. Hanzo, "Iterative joint channel estimation and multi-user detection for multiple-antenna aided OFDM systems," IEEE Trans. Wireless Commun., vol. 6, no. 8, pp. 2904-2914, Aug. 2007.

[6] D. K. C. So and R. S. Cheng, "Iterative EM receiver for space-time coded systems in MIMO frequency-selective fading channels with channel gain and order estimation," IEEE Trans. Wireless Commun., vol. 3, no. 6, pp. 1928-1935, Nov. 2004.

[7] A. Assra, W. Hamouda, and A. Youssef, "EM-based joint channel estimation and data detection for MIMO-CDMA systems," IEEE Trans. Veh. Technol., vol. 59, no. 3, pp. 1205-1216, Mar. 2010.

[8] A. Dempster, N. Laird, and D. B. Rubin, "Maximum likelihood from incomplete data via the EM algorithm," J. R. Stat. Soc. Ser. B (Methodological), vol. 39, no. 1, pp. 1-38, 1977.

[9] J. Zheng and B. Rao, "LDPC-coded MIMO systems with unknown block fading channels: Soft MIMO detector design, channel estimation, and code optimization," IEEE Trans. Signal Process., vol. 54, no. 4, pp. 15041518, Apr. 2006.

[10] X. Y. Zhang, D. G. Wang, and J. B. Wei, "Joint symbol detection and channel estimation for MIMO-OFDM systems via the variational Bayesian EM algorithm," in Proc. IEEE WCNC, 2008, pp. 13-17.

[11] J. Ylioinas and M. Juntti, "Iterative joint detection, decoding, and channel estimation in turbo coded MIMO-OFDM," IEEE Trans. Veh. Technol., vol. 58, no. 4, pp. 1784-1796, May 2009.
[12] S. Chen and Y. Wu, "Maximum likelihood joint channel and data estimation using genetic algorithms," IEEE Trans. Signal Process., vol. 46, no. 5, pp. 1469-1473, May 1998.

[13] K. Yen and L. Hanzo, "Genetic algorithm assisted joint multiuser symbol detection and fading channel estimation for synchronous CDMA systems," IEEE J. Sel. Areas Commun., vol. 19, no. 6, pp. 985-998, Jun. 2001.

[14] M. Abuthinien, S. Chen, and L. Hanzo, "Semi-blind joint maximum likelihood channel estimation and data detection for MIMO systems," IEEE Signal Process. Lett., vol. 15, pp. 202-205, 2008.

[15] S. Boyd and L. Vandenberghe, Convex Optimization. Cambridge, U.K.: Cambridge Univ. Press, 2004.

[16] S. Chen, X. Wang, and C. Harris, "Experiments with repeating weighted boosting search for optimization in signal processing applications," IEEE Trans. Syst., Man, Cybern. B, Cybern., vol. 35, no. 4, pp. 682-693, Aug. 2005.

[17] T. Hanne, "On the convergence of multiobjective evolutionary algorithms," Eur. J. Oper. Res., vol. 117, no. 3, pp. 553-564, Sep. 1999.

[18] X. Ma, H. Kobayashi, and S. Schwartz, "An EM-based channel estimation algorithm for space-time and space-frequency block coded OFDM," in Proc. IEEE ICASSP, 2003, vol. 4, pp. IV-389-IV-392.

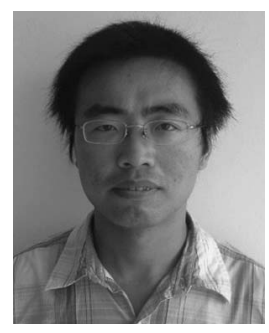

Jiankang Zhang (S'08) received the B.S. degree in mathematics and applied mathematics from the Beijing University of Posts and Telecommunications, Beijing, China, in 2006. He is currently working toward the Ph.D. degree with Zhengzhou University, Zhengzhou, China.

From September 2009 to September 2011, he was a Visiting Student with the School of Electronics and Computer Science, University of Southampton, Southampton, U.K. His research interests are in the areas of wireless communications and signal processing, including space-division multiple access, orthogonal frequency-division multiplexing, channel estimation, and multiuser detection.

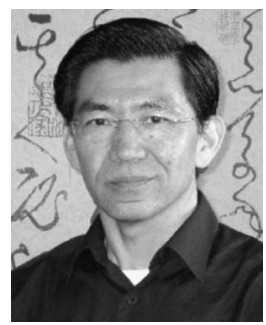

Sheng Chen (M'90-SM-'97-F'08) received the B.Eng. degree in control engineering from the East China Petroleum Institute, Dongying, China, in 1982, the Ph.D. degree in control engineering from the City University, London, U.K., in 1986, and the D.Sc. degree from the University of Southampton, Southampton, U.K., in 2005.

From 1986 to 1999, he held research and academic appointments with the Universities of Sheffield, Edinburgh, and Portsmouth, all in U.K. Since 1999, he has been with the School of Electronics and Computer Science, University of Southampton. His recent research interests include adaptive signal processing, wireless communications, modeling and identification of nonlinear systems, neural network and machine learning, intelligent control system design, evolutionary computation methods, and optimization. He has published over 450 research papers. In the database of the world's most highly cited researchers in various disciplines, compiled by the Institute for Scientific Information, he is on the list of the highly cited researchers in the engineering category; see http://www.ISIHighlyCited.com.

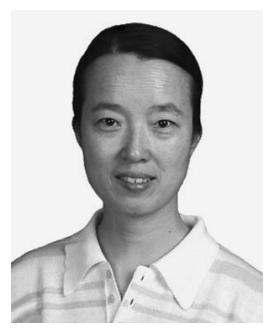

Xiaomin Mu received the M.S. degree from the Beijing Institute of Technology, Beijing, China, in 1982.

She is currently a Full Professor with the School of Information Engineering, Zhengzhou University, Zhengzhou, China. She has published various papers in the field of signal processing and coauthored two books. Her research interests include signal processing in communications systems, wireless communications, and cognitive radio. 


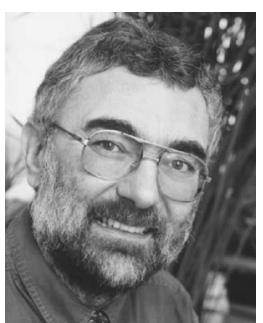

Lajos Hanzo (M'91-SM'92-F'04) received the degree in electronics, the doctorate degree, and the honorary doctorate "Doctor Honaris Causa" degree from the Technical University of Budapest, Budapest, Hungary, in 1976, 1983 and 2009, respectively.

During his 35-year career in telecommunications, he has held various research and academic posts in Hungary, Germany, and the U.K. Since 1986, he has been with the School of Electronics and Computer Science, University of Southampton, Southampton, U.K., where he holds the Chair in telecommunications. He has coauthored 20 John Wiley/IEEE Press books on mobile radio communications totaling in excess of 10000 pages, published in excess of 1000 research entries on IEEE Xplore, acted as Technical Program Committee Chair of IEEE conferences, presented keynote lectures, and been awarded a number of distinctions. He is currently directing an academic research team, working on a range of research projects in the field of wireless multimedia communications sponsored by industry, the Engineering and Physical Sciences Research Council (EPSRC) U.K., the European IST Programme, and the Mobile Virtual Centre of Excellence (VCE), U.K. He is an enthusiastic supporter of industrial and academic liaison and offers a range of industrial courses.

Dr. Hanzo is also a Governor of the IEEE Vehicular Technology Society. Since 2008, he has been the Editor-in-Chief of the IEEE Press and, since 2009, a Chaired Professor, also at Tsinghua University, Beijing, China. 